\title{
Gauss's Law for Magnetism \& Law of Universal Magnetism: Calculate the Charge of a Monopole
}

\author{
Greg Poole \\ Industrial Tests, Inc., Rocklin, CA, USA \\ Email: greg@indtest.com
}

How to cite this paper: Poole, G. (2018) Gauss's Law for Magnetism \& Law of Universal Magnetism: Calculate the Charge of a Monopole. Journal of High Energy Physics, Gravitation and Cosmology, 4, 581-587. https://doi.org/10.4236/jhepgc.2018.44035

Received: April 12, 2018

Accepted: August 5, 2018

Published: August 8, 2018

Copyright $\odot 2018$ by author and Scientific Research Publishing Inc. This work is licensed under the Creative Commons Attribution International License (CC BY 4.0).

http://creativecommons.org/licenses/by/4.0/

\section{Open Access}

\begin{abstract}
This paper describes a mathematical proof that Gauss's Law for Magnetism can be derived from the Law of Universal Magnetism [1]. A second reciprocal proof also shows that the Law of Universal Magnetism can be derived from Gauss's Law for Magnetism. These two complimentary proofs confirm that the Law of Universal Magnetism is a valid equation rooted in Gaussian law. The paper also confirms the theoretical existence of the magnetic monopole and calculates its magnetic charge using the ratio of the electromagnetic field and the speed of light. Using the mass-to-charge ratio of an electron, the mass and radius of the magnetic monopole are determined. The monopole is found to have the same radius as the electron and can also be found in the electromagnetic spectrum range known as gamma rays. Lightning is a natural source of gamma rays and could prove fruitful in the search for monopoles.
\end{abstract}

\section{Keywords}

Gauss's Law for Magnetism, Law of Universal Magnetism, Magnetic Charge Density, Permeability, Monopole

\section{Introduction}

The Law of Universal Magnetism was recently formulated and published earlier this year [1]. In this paper, a proof is offered to determine if the law aligns with Gauss's law for magnetism. Gauss's law for magnetism is a physical application of Gauss's theorem, also known as the divergence theorem in calculus, which was independently discovered by Lagrange in 1762, Gauss in 1813, Ostrogradsky in 1826 , and Green in 1828 [2].

Gauss's law for magnetism is one of the four Maxwell's equations that underlie classical electrodynamics. It states that the magnetic field B has divergence equal to zero [3], is a solenoidal vector field and is equivalent to the statement that magnetic monopoles do not exist [4]. Rather than magnetic charges, the ba- 
sic entity for magnetism is the magnetic dipole. If magnetic monopoles exist, then Gauss's law for magnetism would state that the divergence of $B$ would be proportional to the magnetic charge density $\rho_{m}$, analogous to Gauss's law for electric field. If magnetic monopoles do not exist, then for zero net magnetic charge density $\left(\rho_{m}=0\right)$, the original form of Gauss's magnetism law, is the result. If monopoles are ever found, Gauss's law would have to be modified, as elaborated in this paper.

It was pointed out by Pierre Curie in 1894 that magnetic monopoles could theoretically exist, despite not having been seen so far [5]. Physicist Paul A. M. Dirac, in 1931, proposed the quantum theory of magnetic charge in his paper, Dirac showed that if any magnetic monopoles exist in the universe, then all electric charge in the universe must be quantized [6].

\section{Deriving Gauss's Law for Magnetism from the Law of Universal Magnetism}

Gauss's law for magnetism cannot be derived from Law of Universal Magnetism alone since the Law of Universal Magnetism gives the magnetic field due to an individual magnetic charge only. However, Gauss's law for magnetism can be proven from Law of Universal Magnetism if it is assumed, in addition, that the magnetic field obeys the superposition principle. The superposition principle says that the resulting field is the vector sum of fields generated by each particle or the integral if the magnetic charges are distributed smoothly in space.

Law of Universal Magnetism

$$
F=\frac{k_{e} H_{1} H_{2}}{r^{2} \epsilon}
$$

Substituting the speed of light squared for specific orbital energy $(\epsilon)$ we obtain

$$
F=\frac{k_{e} H_{1} H_{2}}{r^{2} c^{2}}
$$

where,

$F=$ force (Newtons)

$H_{1}$ and $H_{2}=$ magnitudes of magnetic poles (ampere-meter)

$\mu=$ permeability of the intervening medium (tesla meter/ampere, henry/meter or newton/ampere $\left.{ }^{2}\right)$

$$
\begin{aligned}
& r=\text { separation }(\text { meter }) \\
& c^{2}=\text { speed of light }\left(\mathrm{m}^{2} / \mathrm{s}^{2}\right) \\
& k_{e}=c^{2} \mu / 4 \pi
\end{aligned}
$$

Substituting in $\frac{\mu_{0}}{4 \pi}$ for Coulombs Constant $\frac{k_{e}}{c^{2}}$, we obtain the classic force of attraction equation between two magnetic poles when both poles are small enough to be represented as point magnetic charges,

$$
F=\frac{\mu H_{1} H_{2}}{4 \pi r^{2}}
$$

Law of Universal Magnetism states that the magnetic field due to a stationary 
magnetic charge is:

$$
B(r)=\frac{\mu q_{m} e_{r}}{4 \pi r^{2}}
$$

where,

$e_{r}$ is the radial unit vector,

$q_{m}$ is the magnetic charge of a test particle (SI unit: ampere-meter),

$\mu$ is the permeability of the intervening medium (SI unit: tesla meter per ampere, henry per meter or newton per ampere squared),

$r$ is the separation (SI unit: meter).

Using the Law of Universal Magnetism, we get the total field at $r$ by using an integral to sum the field at $r$ due to the infinitesimal change at each other point $s$ in space to give:

$$
B(r)=\frac{\mu}{4 \pi} \int \frac{\rho_{m}(s)(r-s)}{|r-s|^{3}} \mathrm{~d}^{3} s
$$

where $\rho_{m}$ is the magnetic charge density. If we take the divergence of both sides of this equation with respect to $r$ and use the known theorem [7]

$$
\nabla \cdot\left(\frac{r}{|r|^{3}}\right)=4 \pi \delta(r)
$$

where $\delta(r)$ is the Dirac delta function, the result is

$$
\nabla \cdot B(r)=\int \rho(s) \delta(r-s) \mathrm{d}^{3} s
$$

Using the sifting property of the Dirac delta function, we arrive at

$$
\nabla \cdot B(r)=\mu \rho_{m}
$$

where $\mu=\mu_{0}$ the vacuum permeability,

$$
\nabla \cdot B(r)=\mu_{0} \rho_{m}
$$

which is the differential form of Gauss's law for magnetism.

\section{Deriving Law of Universal Magnetism from Gauss's Law for Magnetism}

The Law of Universal Magnetism can be proven from Gauss's law for magnetism if it is assumed, in addition to the curl of the $B$ field, that the magnetic field from a magnetic charge is spherically symmetric. This assumption, like the Law of Universal Magnetism itself, is exactly true if the magnetic charge is stationary, and approximately true if the charge is in motion.

Taking $S$ in the integral form of Gauss' law of magnetism to be a spherical surface of radius $r$, centered at the magnetic charge $q_{m}$ we have

$$
\oint_{s} B \times \mathrm{d} A=\mu q_{m}
$$

By the assumption of spherical symmetry, the integrand is a constant which can be taken out of the integral. The result is

$$
4 \pi r^{2} \hat{r} \cdot B(r)=\mu q_{m}
$$


where $\hat{r}$ is a unit vector pointing radially away from the charge. Again, by spherical symmetry, $B$ points in the radial direction, and so we get

$$
B(r)=\frac{\mu q_{m} e_{r}}{4 \pi r^{2}}
$$

which is essentially equivalent to the Law of Universal Magnetism. Thus the inverse-square law dependence of the magnetic field in the Law of Universal Magnetism follows from Gauss's law for magnetism.

\section{Maxwell's Equation}

Maxwell's equations of electromagnetism relate the electric and magnetic fields to each other and to the motions of electric charges. The standard equations provide for electric charges, but they posit no magnetic charges. Except for this difference, the equations are symmetric under the interchange of the electric and magnetic fields. In fact, symmetric Maxwell's equations can be written when all charges and hence electric currents are zero, and this is how the electromagnetic wave equation is derived.

Fully symmetric Maxwell's equations can also be written if one allows for the possibility ofmagnetic charges analogous to electric charges [8]. With the inclusion of a variable for the density of these magnetic charges $\rho_{m}$, there is also a magnetic current densityvariable in the equations, $j_{m}$.

If magnetic charges do not exist-or if they do exist, but are not present in a region of space-then the new terms in Maxwell's equations are all zero, and the extended equations reduce to the conventional equations of electromagnetism such as

$$
\nabla \cdot B=0
$$

where $\nabla=$ divergence and

$B=$ the magnetic $B$ field.

In the equations in Table $1, \rho_{m}$ is the magnetic charge density, $j_{m}$ is the magnetic current density, and $q_{m}$ is the magnetic charge of a test particle, all defined analogously to the related quantities of electric charge and current; $v$ is the particle's velocity and $c$ is the speed of light.

\section{Calculate Magnetic Charge of a Monopole}

Using the ratio of Gauss's law and Gauss's law of magnetism we obtain,

$$
\frac{\nabla \cdot E}{\nabla \cdot B}=\frac{4 \pi \rho_{e}}{4 \pi \rho_{m}}
$$

The ratio of the electric to magnetic fields in an electromagnetic wave in free space is equal to the speed of light,

$$
c=\frac{\rho_{e}}{\rho_{m}}
$$

Calculate the magnetic charge density, 
Table 1. Maxwell's equations and Lorentz force equation with magnetic monopoles (Gaussian cgs units) [9].

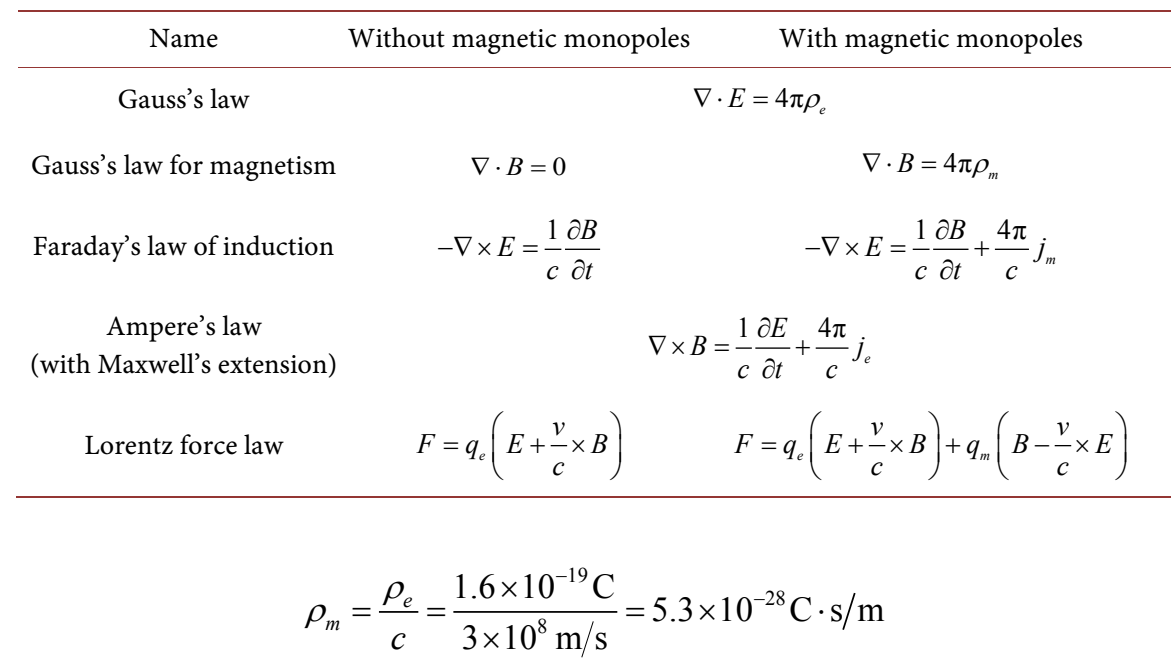

The electron mass-to-charge ratio $(m / Q)$ is a physical quantity that is most widely used in the electrodynamics of charged particles. The mass-to-charge ratio for a magnetic monopole is not known so we will be using the mass-to-charge ratio of the electron for the calculations that follow. The 2014 CODATA recommended value for an electron is $e / m_{e}=-1.758820024 \mathrm{C} / \mathrm{kg}$ [10].

Assuming the monopole is the magnetic charge and mass portion of an electron then the monopole mass is equal to,

$$
\begin{aligned}
& \text { Monopole mass }=\frac{5.3 \times 10^{-28} \mathrm{C} \cdot \mathrm{s} / \mathrm{m}}{1.76 \times 10^{11} \mathrm{C} / \mathrm{kg}} \\
& \text { Monopole mass }=3.0 \times 10^{-39} \mathrm{~kg} \cdot \mathrm{s} / \mathrm{m}
\end{aligned}
$$

Conversion factor from SI unit to Natural Units for momentum,

$$
1 \mathrm{GeV}=5.39 \times 10^{-19} \mathrm{~kg} \cdot \mathrm{m} / \mathrm{s}
$$

Convert to $\mathrm{GeV}$ units,

$$
\begin{aligned}
& \text { Monopole Mass }=\frac{3.0 \times 10^{-39} \mathrm{~kg} \cdot \mathrm{s} / \mathrm{m}}{5.39 \times 10^{-19} \mathrm{~kg} \cdot \mathrm{m} / \mathrm{s}} \\
& \text { Monopole Mass }=5.56 \times 10^{-21} \mathrm{GeV} / \mathrm{c}^{2}
\end{aligned}
$$

Solving for kilograms where,

$$
1 \mathrm{GeV} / \mathrm{c}^{2}=1.783 \times 10^{-27} \mathrm{~kg}
$$

We obtain,

$$
\text { Monopole Mass }=9.9 \times 10^{-48} \mathrm{~kg}
$$

As a point of comparison, the mass of an electron is $9.1 \times 10^{-31} \mathrm{~kg}$.

To determine the size of the magnetic monopole we can substitute the monopole charge and mass into the classical electron radius calculation: 


$$
\begin{gathered}
r_{e}=\frac{1}{4 \pi \epsilon_{0}} \frac{e^{2}}{m_{e} c^{2}} \\
r_{m}=2.8 \times 10^{-15} \mathrm{~m}
\end{gathered}
$$

In comparison the electron has the same radius of the magnetic monopole at $2.8 \times 10^{-15}$ meters. This calculation confirms the assumption of Giacomelli and Patrizil regarding the properties of magnetic monopoles where their paper states the radius of the monopole and electron are the same [11].

Doubling the radius, we obtain a wavelength of $5.6 \times 10^{-15}$ meters, which equates to $v=f=5.35 \times 10^{22} \mathrm{~Hz}$. Using the Plank Hypothesis equation of $E=h v$, we obtain a quantum energy of a magnetic monopole to be $2.2 \times 10^{8} \mathrm{eV}$, which equates to $0.22 \mathrm{GeV}$. The low wavelength and high frequency makes monopoles a condition of gamma rays. Natural sources of gamma rays on Earth are observed in the gamma decay of radionuclides and secondary radiation from atmospheric interactions with cosmic ray particles. There are other terrestrial natural sources, such as lightning strikes and terrestrial gamma-ray flashes.

\section{Conclusions}

A magnetic monopole is a hypothetical elementary particle in particle physics that is an isolated magnet with only one magnetic pole. A magnetic monopole would have a net magnetic charge, either plus or minus. Maxwell's original equations, do not allow magnetic monopoles. However, when Maxwell's equations are rewritten with magnetic charge included, the existence of monopoles is consistent with the updated equation. Modern interest in the concept stems from particle theories, notably the grand unified and superstring theories, which predict their existence.

In this paper we have written a proof whereby Gauss's Law for Magnetism is derived from the Law of Universal Magnetism. A second proof was also written proving the reciprocal, that the Law of Universal Magnetism can be derived from Gauss's Law for Magnetism. The two reciprocal proofs confirm the validity of the Law of Universal Magnetism and also provide proof of the existence of the magnetic monopole. The Law of Universal Magnetism and monopoles are mutually dependent.

Based on the size of the magnetic monopole radius we have concluded that they can be found at or near the frequency range of gamma rays. Lightning is a natural source of high frequency gamma rays and an excellent place to search for magnetic monopoles. Simulating a large short circuit or fault condition to simulate lightning in an electrical laboratory should also result in the production of monopoles. This paper suggests that where there are large quantities of electrons we should find free monopoles as well.

\section{Acknowledgements}

Author wishes to acknowledge ASK Scientific (https://www.askscientific.com) for formatting assistance. Heartfelt thanks to JHEPGC and their editorial staff 
for giving me the opportunity to publish my papers.

\section{References}

[1] Poole, G. (2018) Law of Universal Magnetism. JHEPGC, In Press.

[2] Katz, V.J. (1979) The History of Stokes's Theorem. Mathematics Magazine. Mathematical Association of America, 52, 146-156.

[3] Chow, T.L. (2006) Electromagnetic Theory: A Modern Perspective. Jones and Bartlett, Boston, 134.

[4] Jackson, J.D. (1999) Classical Electrodynamics. 3rd Edition, Wiley, Hoboken, 237.

[5] Curie, P. (1894) Sur la possibilitéd'existence de la conductibilitémagnétique et du magnétismelibre [On the Possible Existence of Magnetic Conductivity and Free Magnetism.] Séances de la Société Française de Physique, Paris, 76.

[6] Dirac, P. (1931) Quantised Singularities in the Electromagnetic Field. Proceedings of the Royal Society $A$ (London), 133, 60.

[7] Griffiths, D.J. (2013) Introduction to Electrodynamics. 4th Edition, Prentice Hall, Upper Saddle River, 50.

[8] http://www.ieeeghn.org/wiki/index.php/STARS:Maxwell\%27s_Equations

[9] Nowakowski, M. and Kelkar, N.G. (2005) Faradays Law in the Presence of Monopoles. Europhysics Letters, 71, 346. arXiv:physics/0508099 [physics.class-ph]

[10] IUPAC (1997) Compendium of Chemical Terminology. 2nd Edition, the "Gold Book". Online Corrected Version: (2006-) "Mass-to-Charge Ratio, m/z in Mass Spectrometry".

[11] Giacomelli, G. and Patrizii, L. (2002) Magnetic Monopole Searches. Dipartimento di Fisica dell'Universit 'a di Bologna and INFN, Sezione di Bologna, Summer School on Astroparticle Physics and Cosmology ICTP, Trieste, 17 June-5 July 2002. 